\title{
The Rule of Hessenberg Matrix for Computing the Determinant of Centrosymmetric Matrices
}

\author{
Nur Khasanah¹, Agustin Absari Wahyu Kuntarini ${ }^{2}$ \\ 1,2Department of Mathematics, Faculty of Science and Technology UIN Walisongo \\ Semarang \\ Email: khasanah.nur@walisongo.ac.id
}

\begin{abstract}
The application of centrosymmetric matrix on engineering takes their part, particularly about determinant rule. This basic rule needs a computational process for determining the appropriate algorithm. Therefore, the algorithm of the determinant kind of Hessenberg matrix is used for computing the determinant of the centrosymmetric matrix more efficiently. This paper shows the algorithm of lower Hessenberg and sparse Hessenberg matrix to construct the efficient algorithm of the determinant of a centrosymmetric matrix. Using the special structure of a centrosymmetric matrix, the algorithm of this determinant is useful for their characteristics.
\end{abstract}

Key Words : Hessenberg; Determinant; Centrosymmetric

\section{INTRODUCTION}

One of the widely used studies in the use of centrosymmetric matrices is how to get determinant from centrosymmetric matrices. Besides this special matrix has some applications [1], it also has some properties used for determinant purpose [2]. Special characteristic centrosymmetric at this entry is evaluated at [3] resulting in the algorithm of centrosymmetric matrix at determinant. Due to sparse structure of this entry, the evaluation of the determinant matrix has simpler operations than full matrix entries.

One special sparse matrix having rules on numerical analysis and arise at centrosymmetric the determinant matrix is the Hessenberg matrix. The role of Hessenberg matrix decomposition is the important role of computing the eigenvalue matrix. In the discussion, the recursive algorithm is explained to compute the $n$-per-n determinant of the Hessenberg matrix [4]. This study is the evaluation of some researches before about the determinant Hessenberg matrix $[5,6]$.

The rule of the Hessenberg matrix for computing the determinant of general centrosymmetric matrix based on block matrix has been done by [7]. Moreover, this study continued by [8] on evaluating the previous work and shows only lower Hessenbeg matrix as block centrosymmetric matrix can use this algorithm. Then necessary and sufficient condition for this algorithm is constructed also that can be evaluated for the determinant process [9]. A Numerical example also shows for more understanding at a special centrosymmetric matrix on its block.

Furthermore, the determinant of sparse Hessenberg matrix is also constructed for resulting in the new algorithm for a pentadiagonal matrix[10]. Some studies are also 
focussing on the determinant algorithm for computational process [11-17]. Based on the previous study, we propose a new form of pentadiagonal matrix with construction entries based on a centrosymmetric matrix called pentadiagonal centrosymmetric matrix. We then continue applying the previous algorithm to compute our new form of pentadiagonal centrosymmetric matrix for determinant purpose. Finally, this paper shows two algorithms of centrosymmetric matrix determinant by using the algorithm of the determinant of the Hessenberg matrix.

\section{PRELIMINARIES}

Before we discuss the main result, let introduce some definitions and properties used to explain the determinant of centrosymmetric matrix using Hessenberg's determinant.

Definition 1 [3] The form of $n$-by- $n$ lower Hessenberg matrix is written as the matrix with the entries as follow

$$
\mathbf{H}=\left(\begin{array}{ccccc}
h_{11} & h_{1,2} & 0 & 0 & 0 \\
h_{21} & h_{2,2} & h_{2,3} & 0 & 0 \\
\vdots & \vdots & \ddots & \ddots & 0 \\
h_{n-1,1} & h_{n-1,2} & \cdots & h_{n-1, n-1} & h_{n-1, n} \\
h_{n, 1} & h_{n, 2} & \cdots & h_{n, n-1} & h_{n, n}
\end{array}\right) .
$$

Definition 2 [10] The sparse Hessenberg matrix with order $n$ is the matrix with construction as

$$
\mathbf{S}=\left(\begin{array}{ccccccc}
h_{11} & h_{1,2} & h_{1,3} & & & & \\
h_{21} & h_{2,2} & h_{2,3} & h_{2,4} & & & \\
& h_{3,2} & h_{3,3} & h_{3,4} & h_{3,5} & & \\
& & \ddots & \ddots & \ddots & \ddots & \\
& & & \ddots & \ddots & \ddots & h_{n-2, n} \\
& & & & h_{n-1, n-2} & h_{n-1, n-1} & h_{n-1, n} \\
& & & & & h_{n, n-1} & h_{n, n-}
\end{array}\right) .
$$

Definition 3 [2] Let $\mathbf{A}=\left(a_{i j}\right)_{n \times n} \in R^{n \times n}$ be a centrosymmetric matrix, if the entries of the matrix satisfy $a_{i j}=a_{n-i+1, n-j+1}$ for $1 \leq i \leq n, 1 \leq j \leq n$ or

$$
\mathbf{A}=\left(\begin{array}{cccc}
a_{11} & a_{12} & \cdots & a_{1, n} \\
a_{21} & a_{22} & \cdots & a_{2, n} \\
\vdots & \vdots & \vdots & \vdots \\
a_{2, n} & \cdots & a_{22} & a_{21} \\
a_{1, n} & \cdots & a_{12} & a_{11}
\end{array}\right)
$$


Definition 4 [7] The centrosymmetric matrix has a special structure that can be constructed as $\mathbf{J}_{\mathbf{n}} \mathbf{A} \mathbf{J}_{\mathbf{n}}=\mathbf{A}$, where $\mathbf{J}_{\mathbf{n}}=\left(\mathbf{e}_{\mathbf{n}}, \mathbf{e}_{\mathbf{n}-\mathbf{1}}, \cdots, \mathbf{e}_{\mathbf{1}}\right)$ and $\mathbf{e}_{\mathbf{i}}$ is the unit vector with the $i$-th element 1 and others 0 .

Definition 5 [8] The centrosymmetric matrix with $\mathrm{n}$ order where $n=2 m$ is even number order can be partitioned with the form

$$
\mathbf{A}=\left(\begin{array}{l|l|l}
\mathbf{B} & \mathbf{J}_{\mathbf{m}} \mathbf{C} \mathbf{J}_{\mathrm{m}} \\
\hline \mathbf{C} & \mathbf{J}_{\mathbf{m}} \mathbf{B} \mathbf{J}_{\mathrm{m}}
\end{array}\right) .
$$

\section{RESULTS AND DISCUSSION}

This part shows the rule of the determinant of lower Hessenberg and sparse Hessenberg matrix for determinant concept of the centrosymmetric matrix. Further discussion is also given for explaining the result and deeper understanding.

\section{Determinant Lower Hessenberg Matrix for Centrosymmetric Matrix's Determinant}

The algorithm of the determinant of lower Hessenberg is presented based on the definition and properties of this matrix. The following is the Lemma of the determinant of lower Hessenberg, which is constructed as a block matrix.

Lemma 6 [7] Let $\tilde{\mathbf{H}}=\left(\begin{array}{c|c}\mathbf{e}_{1}^{\mathbf{T}} & 0 \\ \hline \mathbf{H} & \mathbf{e}_{\mathbf{n}}\end{array}\right)=\left(\begin{array}{ccccc|c}1 & 0 & \cdots & \cdots & 0 & 0 \\ \hline h_{11} & h_{1,2} & 0 & 0 & 0 & 0 \\ h_{21} & h_{2,2} & h_{2,3} & 0 & 0 & \vdots \\ \vdots & \vdots & \ddots & \ddots & 0 & \vdots \\ h_{n-1,1} & h_{n-1,2} & \cdots & h_{n-1, n-1} & h_{n-1, n} & 0 \\ h_{n, 1} & h_{n, 2} & \cdots & h_{n, n-1} & h_{n, n} & 1\end{array}\right)$ is the partition matrix and $\widetilde{\mathbf{H}}=\left(\begin{array}{c|c}\mathbf{e}_{1}^{\mathbf{T}} & 0 \\ \hline \mathbf{H} & \mathbf{e}_{\mathbf{n}}\end{array}\right)=\left(\begin{array}{ccccc|c}1 & 0 & \cdots & \cdots & 0 & 0 \\ \hline h_{11} & h_{1,2} & 0 & 0 & 0 & 0 \\ h_{21} & h_{2,2} & h_{2,3} & 0 & 0 & \vdots \\ \vdots & \vdots & \ddots & \ddots & 0 & \vdots \\ h_{n-1,1} & h_{n-1,2} & \cdots & h_{n-1, n-1} & h_{n-1, n} & 0 \\ h_{n, 1} & h_{n, 2} & \cdots & h_{n, n-1} & h_{n, n} & 1\end{array}\right)$ as the inverse matrix of its matrix, then the determinant of this matrix is written as $\operatorname{det}(\mathbf{H})=(-1)^{n} h \cdot \prod_{i=1}^{n-1} h_{i, i+1}$.

This algorithm is used for evaluating the algorithm of the determinant of centrosymmetric matrix with lower Hessenberg as a block matrix. The algorithm of the determinant of the centrosymmetric matrix with lower Hessenberg as block matrix can be proposed as follow.

$$
\text { Input : Matrix } \mathbf{A}=\left(\begin{array}{l|l}
\mathbf{B} & \mathbf{J}_{\mathrm{m}} \mathbf{C \mathbf { J } _ { \mathrm { m } }} \\
\hline \mathbf{C} & \mathbf{J}_{\mathrm{m}} \mathbf{B} \mathbf{J}_{\mathrm{m}}
\end{array}\right)
$$

Output : $\operatorname{det}(\mathbf{A})$
1) Operate $\mathbf{P}^{\mathrm{T}} \mathbf{A} \mathbf{P}=\left(\begin{array}{c}\mathbf{B}-\mathbf{J}_{\mathbf{m}} \mathbf{C} \\ 0\end{array}\right.$

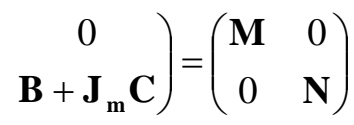


2) Construct $\mathbf{M}, \mathbf{N}$ are Hessenberg matrices become $\tilde{\mathbf{M}}=\left(\begin{array}{cc}\mathbf{e}_{1}^{\mathbf{T}} & 0 \\ \mathbf{M} & \mathbf{e}_{\mathbf{m}}\end{array}\right)$, $\tilde{\mathbf{N}}=\left(\begin{array}{cc}\mathbf{e}_{1}^{\mathbf{T}} & 0 \\ \mathbf{N} & \mathbf{e}_{\mathbf{m}}\end{array}\right)$

3) Let inverse matrices $\tilde{\mathbf{M}}^{-1}=\left(\begin{array}{ll}\boldsymbol{\alpha}_{\mathbf{M}} & \mathbf{L}_{\mathbf{M}} \\ h_{M} & \boldsymbol{\beta}_{\mathbf{M}}^{\mathbf{T}}\end{array}\right), \tilde{\mathbf{N}}^{-1}=\left(\begin{array}{ll}\boldsymbol{\alpha}_{\mathbf{N}} & \mathbf{L}_{\mathbf{N}} \\ h_{N} & \boldsymbol{\beta}_{\mathbf{N}}^{\mathbf{T}}\end{array}\right)$

4) Compute $\operatorname{det}(\mathbf{H})=\operatorname{det}(\mathbf{P}) \cdot \operatorname{det}\left(\begin{array}{cc}\mathbf{M} & 0 \\ 0 & \mathbf{N}\end{array}\right) \cdot \operatorname{det}\left(\mathbf{P}^{\mathbf{T}}\right)=\operatorname{det}(\mathbf{M}) \cdot \operatorname{det}(\mathbf{N})$

5) $\operatorname{det}(\mathbf{H})=(-1)^{m} h_{M} \cdot \prod_{i=1}^{m-1} g_{i, i+1} \cdot(-1)^{m} h_{N} \cdot \prod_{i=1}^{m-1} q_{i, i+1} \cdot$

Or we can write this algorithm by the following theorem for computing the determinant of centrosymmetric matrix with applying the algorithm of the determinant of lower Hessenberg matrix. This algorithm is only applied on a specific centrosymmetric matrix based on the special block matrix.

Theorem 7 [9] Let centrosymmetric matrix with its block matrix, lower Hessenberg matrix, and its notation, such $\mathbf{H}, \mathbf{P}, \mathbf{M}, \mathbf{N}, \tilde{\mathbf{M}}^{-\mathbf{1}}, \tilde{\mathbf{N}}^{-1}$, then the determinant of the centrosymmetric matrix is written as

$$
\operatorname{det}(\mathbf{H})=h_{N} \cdot h_{M} \cdot \prod_{i=1}^{m-1}\left(g_{i, i+1} \cdot q_{i, i+1}\right)
$$

Proof. From the definition of the centrosymmetric matrix, this matrix can be formed as a block matrix $\mathbf{A}=\left(\begin{array}{c|c|}\mathbf{B} & \mathbf{J}_{\mathbf{m}} \mathbf{C} \mathbf{J}_{\mathbf{m}} \\ \hline \mathbf{C} & \mathbf{J}_{\mathbf{m}} \mathbf{B} \mathbf{J}_{\mathrm{m}}\end{array}\right)$. By the orthogonal matrix $\mathbf{P}=\frac{\sqrt{2}}{2}\left(\begin{array}{cc}\mathbf{I}_{\mathrm{m}} & \mathbf{I}_{\mathrm{m}} \\ -\mathbf{J}_{\mathrm{m}} & \mathbf{J}_{\mathrm{m}}\end{array}\right)$, this block matrix can be constructed as $\mathbf{P}^{\mathrm{T}} \mathbf{H P}=\left(\begin{array}{cc}\mathbf{B}-\mathbf{J}_{\mathbf{m}} \mathbf{C} & 0 \\ 0 & \mathbf{B}+\mathbf{J}_{\mathbf{m}} \mathbf{C}\end{array}\right)=\left(\begin{array}{cc}\mathbf{M} & 0 \\ 0 & \mathbf{N}\end{array}\right)$. Based on this matrix, the determinant of centrosymmetric matrix only calculate on block matrices the determinant. These block matrices $\mathbf{M}, \mathbf{N}$ are Hessenberg form, therefore the algorithm of determinant lower Hessenberg can be applied for this determinant. This step becomes the main necessary for the next algorithm.

By the algorithm of lower Hessenberg matrix, let's form $\tilde{\mathbf{M}}=\left(\begin{array}{cc}\mathbf{e}_{1}^{\mathbf{T}} & 0 \\ \mathbf{M} & \mathbf{e}_{\mathbf{m}}\end{array}\right)$, $\tilde{\mathbf{N}}=\left(\begin{array}{cc}\mathbf{e}_{1}^{\mathbf{T}} & 0 \\ \mathbf{N} & \mathbf{e}_{\mathbf{m}}\end{array}\right)$, and assume $\tilde{\mathbf{M}}^{-\mathbf{1}}=\left(\begin{array}{ll}\boldsymbol{\alpha}_{\mathbf{M}} & \mathbf{L}_{\mathbf{M}} \\ h_{M} & \boldsymbol{\beta}_{\mathbf{M}}^{\mathbf{T}}\end{array}\right), \quad \tilde{\mathbf{N}}^{-1}=\left(\begin{array}{ll}\boldsymbol{\alpha}_{\mathbf{N}} & \mathbf{L}_{\mathbf{N}} \\ h_{N} & \boldsymbol{\beta}_{\mathbf{N}}^{\mathbf{T}}\end{array}\right)$, where $\boldsymbol{\alpha}_{\mathbf{M}, \mathbf{N}}, \mathbf{L}_{\mathbf{M}, \mathbf{N}}$, $\boldsymbol{\beta}_{\mathrm{M}, \mathbf{N}}$ are matrices with the size of $n \times 1, n \times n, n \times 1$ respectively and $h_{M, N}$ is scalar. By analytical process at lower Hessenberg algorithm, then we find $h_{M} \neq 0$ and $h_{N} \neq 0$. Finally, the determinant of the centrosymmetric matrix based on lower Hessenberg form is

$\operatorname{det}(\mathbf{H})=\operatorname{det}(\mathbf{P}) \cdot \operatorname{det}\left(\begin{array}{cc}\mathbf{M} & 0 \\ 0 & \mathbf{N}\end{array}\right) \cdot \operatorname{det}\left(\mathbf{P}^{\mathbf{T}}\right)=\operatorname{det}(\mathbf{M}) \cdot \operatorname{det}(\mathbf{N})$ 


\section{Determinant Sparse Hessenberg Matrix for Centrosymmetric Matrix's Determinant}

Based on the previous study, [10] shows the efficient algorithm general pentadiagonal matrix. Then, this paper gives a specific discussion on the determinant of pentadiagonal centrosymmetric matrix by applying a pentadiagonal determinant matrix. There are some basic definitions for further study on the determinant of pentadiagonal centrosymmetric matrix.

Definition $\left[\mathbf{1 7 , 1 8 ]}\right.$ Let $\mathbf{D}=\left(d_{i j}\right)_{1 \leq i, j \leq n}$ is called $n \times n$ general pentadiagonal matrix which the entry $d_{i j}=0$ for $|i-j|>2$ or it can be constructed as

$\mathbf{D}=\left(\begin{array}{ccccccc}d_{11} & d_{12} & d_{13} & & & & \\ d_{21} & d_{22} & d_{23} & d_{24} & & & \\ d_{31} & d_{32} & d_{33} & d_{34} & d_{35} & & \\ & \ddots & \ddots & \ddots & \ddots & \ddots & \\ & & \ddots & \ddots & \ddots & \ddots & d_{n-2, n} \\ & & & d_{n-1, n-3} & d_{n-1, n-2} & d_{n-1, n-1} & d_{n-1, n} \\ & & & & d_{n, n-2} & d_{n, n-1} & d_{n, n}\end{array}\right)$.

This performance of the general pentadiagonal matrix has been described, and moreover, the algorithm of determinant general pentadiagonal is also evaluated [10]. This paper will give a different point of view from a general structure pentadigonal matrix, which can be constructed as a centrosymmetric matrix. This work combines the general pentadiagonal matrix's definition, which has a centrosymmetric matrix structure, then we call the pentadiagonal centrosymmetric matrix.

Therefore, we construct the algorithm of the pentadiagonal centrosymmetric matrix based on the algorithm of the determinant of the general pentadiagonal matrix. The algorithm has the following steps.

1. Construct pentadiagonal centrosymmetric matrix

Based on the definition of pentadiagonal and centrosymmetric matrix, then the pentadiagonal centrosymmetric matrix is written as

$$
\mathbf{D}=\left(\begin{array}{ccccccc}
d_{11} & d_{12} & d_{13} & & & & \\
d_{21} & d_{22} & d_{23} & d_{24} & & & \\
d_{31} & d_{32} & d_{33} & d_{34} & d_{35} & & \\
& \ddots & \ddots & \ddots & \ddots & \ddots & \\
& & \ddots & \ddots & \ddots & \ddots & d_{n-2, n} \\
& & & d_{n-1, n-3} & d_{n-1, n-2} & d_{n-1, n-1} & d_{n-1, n} \\
& & & & d_{n, n-2} & d_{n, n-1} & d_{n, n}
\end{array}\right)
$$

where the entries have a centrosymmetric structure $a_{i j}=a_{n-i+1, n-j+1}$ for $1 \leq i \leq n, 1 \leq j \leq n$.

2. Transform into sperse Hessenberg matrix

According to the form of sparse Hessenberg matrix, then the construction of sparse Hessenberg matrix $\mathbf{D}=\left(d_{i j}\right)_{1 \leq i, j \leq n}$ is written as 
$\mathbf{H}=\left(\begin{array}{ccccccc}d_{11} & d_{12} & d_{13} & & & & \\ d_{21} & d_{22} & d_{23} & d_{24} & & & \\ & d_{32} & d_{33} & d_{34} & d_{35} & & \\ & & \ddots & \ddots & \ddots & \ddots & \\ & & & \ddots & \ddots & \ddots & d_{n-2, n} \\ & & & & d_{n-1, n-2} & d_{n-1, n-1} & d_{n-1, n} \\ & & & & & d_{n, n-1} & d_{n, n}\end{array}\right)$.

The form of (8) matrix is resulted by choosing $\boldsymbol{\lambda}_{\mathbf{i}+\mathbf{1}, \mathbf{i}}$ from the matrix of

$$
\lambda_{i+1}=\left(\begin{array}{cccc}
I_{i-1} & & & \\
& 1 & & \\
& \lambda_{i+1, i} & 1 & \\
& & & I_{n-i-1}
\end{array}\right) .
$$

For instance, let pentadiagonal centrosymmetric matrix with the size $8 \times 8$ and choose $\lambda_{3,2}=-\frac{d_{3,1}}{d_{2,1}}$ for transforming matrix as follows.

$$
\begin{aligned}
& \lambda_{3} \mathbf{D}=\left(\begin{array}{ccccccccccc}
1 & & & & & & \\
& 1 & & & & & \\
& -\frac{d_{3,1}}{d_{2,1}} & & & & & & & \\
& & & 1 & & & \\
& & & 1 & & & \\
& & & & 1 & & \\
& & & & & 1 & \\
& & & & & & 1
\end{array}\right) \cdot\left(\begin{array}{lllllllll}
d_{11} & d_{12} & d_{13} & & & & & \\
d_{21} & d_{22} & d_{23} & d_{24} & & & & \\
d_{31} & d_{32} & d_{33} & d_{34} & d_{35} & & & \\
& d_{42} & d_{43} & d_{44} & d_{45} & d_{56} & & \\
& & d_{53} & d_{54} & d_{55} & d_{56} & d_{57} & \\
& & & d_{64} & d_{65} & d_{66} & d_{67} & d_{68} \\
& & & & d_{75} & d_{76} & d_{77} & d_{78} \\
& & & & & d_{86} & d_{87} & d_{88}
\end{array}\right) \\
& =\left(\begin{array}{llllllll}
d_{11} & d_{12} & d_{13} & & & & \\
d_{21} & d_{22} & d_{23} & d_{24} & & & & \\
& \tilde{d}_{32} & d_{33} & d_{34} & d_{35} & & & \\
& d_{42} & d_{43} & d_{44} & d_{45} & d_{56} & & \\
& & d_{53} & d_{54} & d_{55} & d_{56} & d_{57} & \\
& & d_{64} & d_{65} & d_{66} & d_{67} & d_{68} \\
& & & d_{75} & d_{76} & d_{77} & d_{78} \\
& & & & & d_{86} & d_{87} & d_{88}
\end{array}\right)
\end{aligned}
$$

By the same way, taking $\lambda_{4,3}=-\frac{d_{42}}{d_{32}}, \cdots, \lambda_{87}=-\frac{d_{86}}{d_{76}}$ and the process will have the following Hessenberg matrix as follow. 


$$
\lambda_{8} \lambda_{7} \lambda_{6} \cdots \lambda_{3} \mathbf{D}=\left(\begin{array}{lllllllll}
d_{11} & d_{12} & d_{13} & & & & & \\
d_{21} & d_{22} & d_{23} & d_{24} & & & & \\
& \tilde{d}_{32} & \tilde{d}_{33} & \tilde{d}_{34} & d_{35} & & & \\
& & \tilde{d}_{43} & \tilde{d}_{44} & \tilde{d}_{45} & d_{56} & & \\
& & & \tilde{d}_{54} & \tilde{d}_{55} & \tilde{d}_{56} & d_{57} & \\
& & & \tilde{d}_{65} & \tilde{d}_{66} & \tilde{d}_{67} & d_{68} \\
& & & & \tilde{d}_{76} & \tilde{d}_{77} & \tilde{d}_{78} \\
& & & & & & \tilde{d}_{87} & \tilde{d}_{88}
\end{array}\right) .
$$

Generally, the formula of the value of $\lambda_{i+1, i}$ is defined as $\lambda_{i+1, i}=-\frac{d_{i+1, i-1}}{\tilde{d}_{i, i-1}}$ with $\tilde{d}_{21}=d_{21}$. Remembering that, $\mathbf{V}=\lambda_{n} \lambda_{n-1} \cdots \lambda_{3}$ is the matrix which formed by $\lambda$ matrix , having the form of $\mathbf{V D}=\mathbf{H}$. Consequently,

$$
\operatorname{det}(\mathbf{D})=\operatorname{det}(\mathbf{V}) \cdot \operatorname{det}(\mathbf{D})=\operatorname{det}(\mathbf{V D})=\operatorname{det}(\mathbf{H}) \text {, where } \operatorname{det}(\mathbf{V})=1
$$

By the entries of $\mathbf{D}$ which integer numbers or $\mathbf{d}_{\mathbf{i j}} \in \mathbf{Z}$, then it can use the following matrix

$$
\lambda_{i+1}=\left(\begin{array}{cccc}
I_{i-1} & & & \\
& 1 & & \\
& -d_{i+1, i-1} & \tilde{d}_{i, i-1} & \\
& & & I_{n-i-1}
\end{array}\right) .
$$

Furthermore, to compute the determinant of the pentadiagonal centrosymmetric matrix is only on computing the determinant of the Hessenberg matrix. Then, the next step is how to compute the Hessenberg matrix.

3. Compute the Hessenberg Matrix

By applying the previous algorithm, this determinant is constructed by using the two-term recurrence. This step takes the following explanation.

Let $n \times n$ matrix $\mathbf{Z}_{\mathbf{n}}=\left(\begin{array}{cc}\mathbf{P}_{\mathbf{n}-\mathbf{1}} & \mathbf{q}_{\mathbf{n}-\mathbf{1}} \\ \mathbf{r}^{\mathrm{T}}{ }_{\mathbf{n}-\mathbf{1}} & \mathbf{S}_{\mathbf{n}}\end{array}\right)$, where the size of block matrices is $\mathbf{Z}_{\mathbf{n}-\mathbf{1}}$ has $(n-1) \times(n-1), \mathbf{q}_{\mathbf{n}-1}, \mathbf{S}_{\mathbf{n}}$ are the scalar and $\mathbf{r}^{\mathbf{T}}{ }_{\mathbf{n}-1}$ has $1 \times(n-1)$. Then the determinant of $\mathbf{Z}_{\mathbf{n}}$ recursively is written as :

$$
\begin{aligned}
& \mathbf{f}_{\mathbf{0}}=1 \\
& \mathbf{f}_{\mathbf{i}}=\boldsymbol{\alpha}_{\mathbf{i}} \mathbf{f}_{\mathbf{i}-\mathbf{1}} \text {, where } \boldsymbol{\alpha}_{\mathbf{1}}=\mathbf{d}_{\mathbf{1}}, \boldsymbol{\alpha}_{\mathbf{i}}=\mathbf{s}_{\mathbf{i}}-\mathbf{r}_{\mathbf{i}-\mathbf{1}}{ }^{\mathrm{T}} \mathbf{Z}_{\mathbf{i}-\mathbf{1}}{ }^{-1} \mathbf{q}_{\mathbf{i}-\mathbf{1}}
\end{aligned}
$$

Then, the determinant of $\mathbf{Z}_{\mathbf{i}}$ matrix is $\operatorname{det}\left(\mathbf{Z}_{\mathbf{i}}\right)=\mathbf{f}_{\mathbf{i}}$, where $i=1,2, \cdots, n$.

Now, we apply the previous algorithm for our sparse Hessenberg matrix, and we have the following recursive.

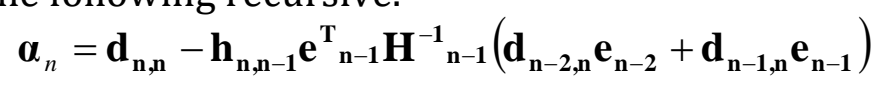




$$
\boldsymbol{\alpha}_{n}=\mathbf{d}_{\mathbf{n}, \mathbf{n}}-\mathbf{d}_{\mathbf{n}, \mathbf{n}-\mathbf{1}}\left(\mathbf{d}_{\mathbf{n}-2, \mathbf{n}} \mathbf{e}^{\mathbf{T}}{ }_{\mathbf{n}-1} \mathbf{H}^{-1}{ }_{n-1} \mathbf{e}_{\mathbf{n}-2}+\mathbf{d}_{\mathbf{n}-\mathbf{1}, \mathbf{n}} \mathbf{e}^{\mathbf{T}}{ }_{\mathbf{n}-1} \mathbf{H}^{-1}{ }_{n-1} \mathbf{e}_{n-1}\right) \text {, where } \mathbf{e} \text { is a }
$$

vector unit.

By substituting the equations $\quad \mathbf{e}^{\mathbf{T}}{ }_{\mathbf{n}-1} \mathbf{H}^{-1}{ }_{\mathbf{n}-1} \mathbf{e}_{n-2}=-\frac{\mathbf{f}_{n-3}}{\mathbf{f}_{\mathbf{n}-\mathbf{1}}} \mathbf{d}_{\mathbf{n}-\mathbf{1}, \mathbf{n}-\mathbf{2}} \quad$ and $\mathbf{e}^{T}{ }_{n-1} \mathbf{H}^{-1}{ }_{n-1} \mathbf{e}_{n-1}=-\frac{\mathbf{f}_{n-2}}{\mathbf{f}_{n-1}}$ then become

$\boldsymbol{\alpha}_{n}=\mathbf{d}_{\mathbf{n}, \mathbf{n}}-\mathbf{d}_{\mathbf{n}, \mathbf{n}-\mathbf{1}}\left(\frac{\mathbf{f}_{\mathbf{n}-\mathbf{3}}}{\mathbf{f}_{\mathbf{n}-\mathbf{1}}} \mathbf{d}_{\mathbf{n}-\mathbf{1}, \mathbf{n}-\mathbf{2}} \mathbf{d}_{\mathbf{n}-\mathbf{2}, \mathbf{n}}-\frac{\mathbf{f}_{\mathbf{n}-\mathbf{2}}}{\mathbf{f}_{\mathbf{n}-\mathbf{1}}} \mathbf{d}_{\mathbf{n}-\mathbf{1}, \mathbf{n}}\right)$. Then, by multiply the equation with

$\mathbf{f}_{n-1}$ we have

$f_{n}=\alpha_{n} f_{n-1}=f_{n-1} d_{n, n}+d_{n, n-1}\left(f_{n-3} d_{n-1, n-2} d_{n-2, n}-f_{n-2} d_{n-1, n}\right)$.

4. Construct the Algorithm of Determinant Pentadiagonal Centrosymmetric Matrix

$$
\begin{aligned}
& \mathbf{f}_{\mathbf{1}}=\mathbf{d}_{\mathbf{1 1}} \\
& \mathbf{f}_{2}=\mathbf{f}_{\mathbf{1}} \mathbf{d}_{\mathbf{2 2}}-\mathbf{d}_{\mathbf{2 1}} \mathbf{d}_{\mathbf{1 2}} \\
& \mathbf{f}_{\mathbf{3}}=\mathbf{f}_{\mathbf{2}} \mathbf{d}_{\mathbf{3 3}}+\mathbf{d}_{\mathbf{3 2}}\left(\mathbf{d}_{\mathbf{2 1}} \mathbf{d}_{\mathbf{1 3}}-\mathbf{f}_{\mathbf{1}} \mathbf{d}_{\mathbf{2 3}}\right) \\
& \quad \text { for } i=4,5, \cdots, n \\
& \quad \mathbf{f}_{i}=f_{i-1} d_{, i}+d_{i, i-1}\left(f_{i-3} d_{i-1, i-2} d_{i-2, i}-f_{i-2} d_{i-1, i}\right)
\end{aligned}
$$

end

$$
\operatorname{det}(\mathbf{D})=\mathbf{f}_{\mathbf{n}} \text {. }
$$

This algorithm shows the rule of the determinant of Hessenberg matrix can be contructed for the general determinant of the pentadiagonal centrosymmetric matrix.

Based on two different algorithms above, the rule of determinant Hesseberg matrix can evaluate the determinant of the centrosymmetric matrix. For efficient algorithm, the first algorithm evaluates the determinant of centrosymmetric matrix applying the algorithm of the determinant of the lower Hessenberg matrix. It happens caused by the lower Hessenberg matrix appear as its block matrix, then the algorithm of centrosymmetric matrix only a half working. On the next algorithm, the algorithm of the sparse Hessenberg matrix is applied to construct the algorithm of determinant general pentadiagonal centrosymmetric matrix. This algorithm is used caused by the same structure of the main matrix is a sparse Hessenberg matrix, therefore is applicable.

\section{CONCLUSIONS}

The different cases of the application of determinant Hesseberg matrix are applied to a different form of a centrosymmetric matrix. It is necessary to identify the form of the main general centrosymmetric from the beginning. This part determines the best algorithm for the computational process more efficient. This paper shows the different Hessenberg appearance on a centrosymmetric matrix results different algorithms of the computing of the determinant. 


\section{REFERENCES}

[1] Datta and Morgera, "On the reducibility of centrosymmetric matrices-Aplication in engineering problems", Circuits System Signal Process., 8 (1) pp. 71-95, 1989.

[2] Zhong-Yun Liu, "Some properties of centrosymmetric matrices", Appl. Math. Comput. 141 pp. 297-306, 2003.

[3] Gene H. Golub and Charles F. Van Loan, "Matrix Computations, third ed.", Johns Hopkins University Press, Baltimore and London, (1996).

[4] Y.-H. Chen, C.-Y. Yu, A new algorithm for computing the inverse and the determinant of a Hessenberg matrix, Appl. Math. Comput. 218 (2011) 44334436.

[5] Tomohiro Sogabe, "On a two-term recurrence for the determinant of a general matrix", Appl. Math. Comput., 187 pp. 785-788, 2007.

[6] Mohamed Elouafi and A.D. Aiat Hadj, "A new recursive algorithm for inverting Hessenberg matrices", Appl. Math. Comput., 214 pp. 497-499, 2009.

[7] Di Zhao and Hongyi Li, "On the computation of inverse and determinant of a kind of special matrices", Appl. Math. Comput., 250 pp. 721-726, 2015.

[8] N Khasanah, Farikhin and B Surarso, "The algorithm of determinant of centrosymmetric matrix based on lower Hessenberg form", IOP Conf. Series : Journal of Physics : Conf. Series $824,2017$.

[9] Nur Khasanah, Bayu Surarso, and Farikhin, "Necessary and sufficient on the computation of determinant of a kind of special matrix", AIP Conference Proceedings 2234, 040014, 2020.

[10] Tomohiro Sogabe, "A fast numerical algorithm for the determinant of a pentadiagonal matrix", Appl. Math. Comput., 196 pp. 835-841, 2008.

[11] Abderraman Marrero and V Tomeo, "Some results on determinants and inverses of nonsingular pentadiagonal matrices", J. Comput. Appl. Math., 275 pp. 447-455, 2015.

[12] Zheng Wang, "The inverse and the determinant of pentadiagonal Toeplitz matrix", IJSM, 5(3) pp. 28-31 2018.

[13] Jiteng Jia, Boting Yang and Sumei Li, “On a homogenous reccurence relation for the determinants of general pentadiagonal Toeplitz matrices", Comp. Math Appl., 71 pp. 1036-1044, 2016.

[14] Jiteng Jia and Sumei Li, "On determinants of cyclic pentadiagonal matrices with Toeplitz structure”, Comp. Math Appl., 73 pp. 304-309, 2017.

[15] Jolanta Borowska, Lena Lacinska and Jowita Rychlewska, "On determinant of certain pentadiagonal matrix”, J. Appl. Math. Comput. Mech., 12(3) pp21-26, 2013.

[16] D.J. Evans, A recursive algorithm for determining the eigenvalues of a quindiagonal matrix, Comput. J. 18 (1973) 70-73.

[17] R.A. Sweet, A recursive relation for the determinant of a pentadiagonal matrix, Comm. ACM 12 (1969) 330-332. 